\title{
HORIZONTAL CELLS IN THE RETINA OF THE RABBIT ${ }^{1}$
}

\author{
RAMON F. DACHEUX ${ }^{2}$ AND ELIO RAVIOLA
}

Department of Anatomy, Harvard Medical School, Boston, Massachusetts 02115

Received March 5, 1982; Revised May 12, 1982; Accepted May 13, 1982

\begin{abstract}
The light responses, morphology, and connections of horizontal cells (HCs) were studied in the retina of the rabbit using intracellular recordings and the injection of visible markers. Two types of HCs were identified, axonless and axon-bearing HCs. Axonless HCs and the somatic end of axonbearing HCs respond to white light of varying intensity with graded hyperpolarizations; both display a transient superimposed on the sustained hyperpolarization at stimulus initiation and a small rod aftereffect at the cessation of high intensity stimuli. Anatomically, both are connected to cones, but their responses also suggest rod influence. Both summate stimuli from a retinal area which is much larger than their respective dendritic fields. However, only axonless HCs transfer a fluorescent, low molecular weight dye to adjoining, homologous cells. The axon terminal of axon-bearing HCs has response properties different from those of the cell body: the transient at stimulus initiation is absent; furthermore, at high levels of illumination, the rod aftereffect becomes equal in amplitude to the primary hyperpolarization. Anatomically, it is connected to rods, but its responses also suggest cone influence. Its receptive field approximates in diameter its anatomical spread and it does not transfer fluorescent dye to its neighbors.
\end{abstract}

The mammalian retina is ideally suited for a combined structural and electrophysiological approach to the problem of the interactions between photoreceptor, horizontal, and bipolar cells. First, the connectivity of the outer plexiform layer in mammals is well understood (Missotten, 1965; Dowling and Boycott, 1966; Kolb, 1970, 1974, 1977; Boycotl and Kolb, 1973). Secondly, the structure of the photoreceptor synapses is typical and the internal organization of the synaptic membranes is known (Raviola and Gilula, 1975). Finally, some, and perhaps all, mammalian bipolar cells make a single type of synaptic junction with the photoreceptors (Kolb, 1970; Boycott and Kolb, 1973) so that the structural features at the synapse can be correlated unequivocally with the postsynaptic response.

So far, the cat has been the animal of choice for intracellular recordings from mammalian retinal neurons (Nelson et al., 1976; Nelson, 1977) and a considerable amount of information exists on the connectivity of the

\footnotetext{
'We thank Ms. Suzanne Kuffler for expert technical assistance and Mr. Peter Ley for help with the photography. This work was supported by United States Public Health Service Grants EY 01344 and EY 03011. R. F. D. is the recipient of New Investigator Research Award EY 03590 .

${ }^{2}$ To whom correspondence should be addressed at Department of Anatomy, Harvard Medical School, 25 Shattuck Street, Boston MA 02115 .
}

outer plexiform layer in this species (Boycott and Kolb, 1973; Kolb, 1974, 1977). However, a superfused rabbit eyecup preparation also was developed (Miller and Dacheux, 1973) and recently modified for electrophysiological studies on the ontogeny of cell communication in the mammalian retina (Dacheux and Miller, 1981a, b). In this simple preparation, the retina remains viable for long periods of time and is optimally preserved for ultrastructural analysis. Therefore, we have selected the isolated rabbit eyecup for a combined anatomical and physiological study of the neural interactions in the outer plexiform layer of the mammalian retina. In this paper, our first results on rabbit horizontal cells are reported: we have found that these neurons have response properties similar to those in the cat (Nelson et al., 1976; Nelson, 1977), thus confirming previous results by Dacheux and Miller (1981a). By intracellular injection of horseradish peroxidase (HRP), we have correlated their response properties with their morphology and traced their synaptic connections with the photoreceptors. Finally, we have analyzed their ability to transfer intracellularly injected Lucifer Yellow CH (LY).

\section{Materials and Methods}

Preparation, stimulation, and recordings. New Zealand albino rabbits weighing 4 to $5 \mathrm{~kg}$ were used. Details of the preparation, light stimulation, and recording techniques were reported elsewhere (Miller and Dacheux, 
1973; Dacheux and Miller, 1981a). Briefly, the eye was enucleated in dim red light under urethane anesthesia $(0.8 \mathrm{gm} / \mathrm{kg})$ and hemisected. The posterior segment was everted and mounted in a shallow chamber so that the vitreal surface of the retina could be exposed to a bicarbonate Ringer's solution flowing at a rate of $20 \mathrm{ml} / \mathrm{min}$. The Ringer's solution contained $120 \mathrm{mM} \mathrm{NaCl}, 5 \mathrm{~mm}$ $\mathrm{KCl}, 25 \mathrm{mM} \mathrm{NaHCO}, 0.8 \mathrm{~mm} \mathrm{Na}_{2} \mathrm{HPO}_{4}, 0.1 \mathrm{~mm}$ $\mathrm{NaH}_{2} \mathrm{PO}_{4}, 1.0 \mathrm{~mm} \mathrm{MgSO}$, $2.0 \mathrm{~mm} \mathrm{CaCl} 2,10 \mathrm{~mm}$ glucose, $56 \mathrm{~nm}$ ascorbic acid, and $1 \%$ fetal calf serum. The perfusate was maintained at a temperature of $35^{\circ}$ to $36^{\circ} \mathrm{C}$ and constantly gassed with an $\mathrm{O}_{2} / \mathrm{CO}_{2}$ mixture to obtain a $\mathrm{pH}$ of $7.4 \pm 0.05$. An interval of less than 3 min elapsed between enucleation and immersion in the incubating medium. The viability of the preparation was controlled by continuously monitoring the electroretinogram (ERG). However, the most sensitive indicator of good viability of the retina was the excellent preservation of its cytological detail when examined with the electron microscope.

The preparation was contained within a light-tight Faraday cage. Light was focused on the retina from tungsten/halogen lamps (100 W; Ealing Corp., South Natick, MA) through the mirrors, prisms, and lenses of a double beam optical system. Maximal irradiance $(0.0$ $\log$ unit) was $6.92 \mathrm{~W} / \mathrm{m}^{2}$ for one beam and $8.03 \mathrm{~W} / \mathrm{m}^{2}$ for the other. Light intensity was controlled by calibrated neutral density filter wheels. The spatial properties of the responses of horizontal cells (HCs) were tested by projecting on the retina spots of light whose diameter varied from $10 \mu \mathrm{m}$ to $6 \mu \mathrm{mm}$. An effort was made to maintain the retina in a relatively dark-adapted state by using a test stimulus intensity $0.5 \mathrm{log}$ unit above the threshold of the ERG $b$ wave during the course of electrode penetration and by prolonging the interval between high intensity stimuli. Intracellular potentials were recorded through a high impedance amplifier equipped with a bridge device for passing current.

Staining and specimen preparation for microscopy. Micropipettes fabricated on a Brown-Flaming puller were filled with $4 \%$ HRP (Grade I, Boehringer Mannheim Biochemicals, Indianapolis, IN) in $0.2 \mathrm{M}$ potassium acetate or 4\% LY (Aldrich Chemical Co., Milwaukee, WI) in $0.1 \%$ lithium chloride (Stewart, 1978). A positive current of 1 to $5 \mathrm{nA}$ was delivered in square pulses of $30 \mathrm{msec}$ duration at a frequency of $15 \mathrm{~Hz}$ for 1 to $6 \mathrm{~min}$ to eject HRP from the electrode. LY was ejected by negative pulses of the same intensity, duration, and frequency. After recording and dye injection, the eyecup was removed from the chamber and immersed in the fixative fluid. HRP-injected specimens were fixed in $2 \%$ formaldehyde, $2.5 \%$ glutaraldehyde in 0.1 M Sörenson's phosphate buffer. The retina was subsequently separated from the sclera, treated with cobalt chloride, and incubated in 3,3',4,4'-biphenyltetra-amine tetrahydrochloride (Matheson Coleman and Bell, Norwood, $\mathrm{OH}$ ) and $\mathrm{H}_{2} \mathrm{O}_{2}$ in cacodylate buffer at $\mathrm{pH} 5.1$ (Adams, 1977). For light microscopy, the retina was dehydrated and mounted in tolo. Both light micrographs and camera lucida drawings were made of the stained cells. For electron microscopy, the retina was postfixed in a mixture of osmium tetroxide and potassium ferrocyanide (Karnovsky, 1971), stained en bloc with uranyl acetate, and cmbedded in Epon/ Araldite. Thin sections were stained with uranyl acetate and lead citrate and examined with a JEOL 100B electron microscope. LY-injected specimens were fixed in $4 \%$ formaldehyde in 0.1 м Sörenson's phosphate buffer, dehydrated, and mounted in Lustrex HF555 (Monsanto Co., St. Louis, MO). Light micrographs were obtained with a Leitz fluorescence microscope equipped with a low bandpass filter (BG 12), a built-in dichroic mirror/filter assembly (TK 510/K515), and a second barrier filter (530 $\mathrm{nm})$.

\section{Results}

Intracellular recordings. Intracellular responses from all identified $\mathrm{HCs}$ in the rabbit retina consisted of graded hyperpolarizations to step changes in the intensity of diffuse illumination. In one class of responses, levels of illumination below ERG $b$ wave threshold (-4.5 log units) produced a slow, low amplitude hyperpolarization with a latency of $34 \mathrm{msec}$. At a stimulus intensity of -3.0 log units, a transient component superimposed on a relatively sustained hyperpolarization was present at the onset of illumination. As the stimulus intensity was increased, the transient component remained, but a small, secondary hyperpolarization, similar to Steinberg's (1969a) rod aftereffect in the cat, appeared after the termination of the stimulus. At a high stimulus intensity of $-1.0 \log$ unit, the response became more rapid and the latency decreased to $10 \mathrm{msec}$; furthermore, the amplitude of the sustained hyperpolarization increased greatly and only slight changes in the rod aftereffect were observed. All of these facts suggest a major input from cones and a weaker influence from rods on the cells generating this class of responses (Fig. 1, $a$ and $b$ ).

Variations in the profile of the response were noted

a.

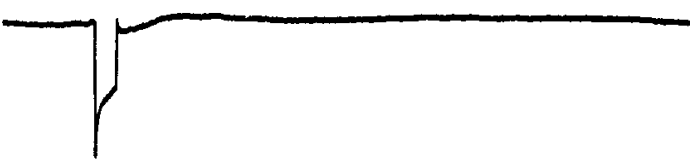

b.
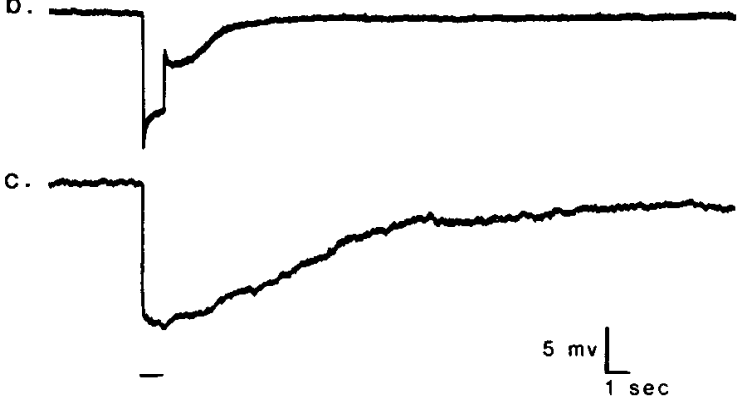

Figure 1. Responses from HCs of the rabbit retina to high intensity stimulation with white light $(-1.0 \mathrm{log}$ unit). The responses from axonless $\mathrm{HCs}(a)$ and from the somatic end of axon-bearing HCs $(b)$ are characterized at the onset of illumination by a transient superimposed on the sustained hyperpolarization; they exhibit a small, secondary hyperpolarization after the termination of the stimulus. The response from the axon terminal of axon-bearing $\mathrm{HCs}(c)$ has no transient at stimulus initiation and is dominated by a prominent rod aftereffect. The presentation of the stimulus is indicated by a bar. 

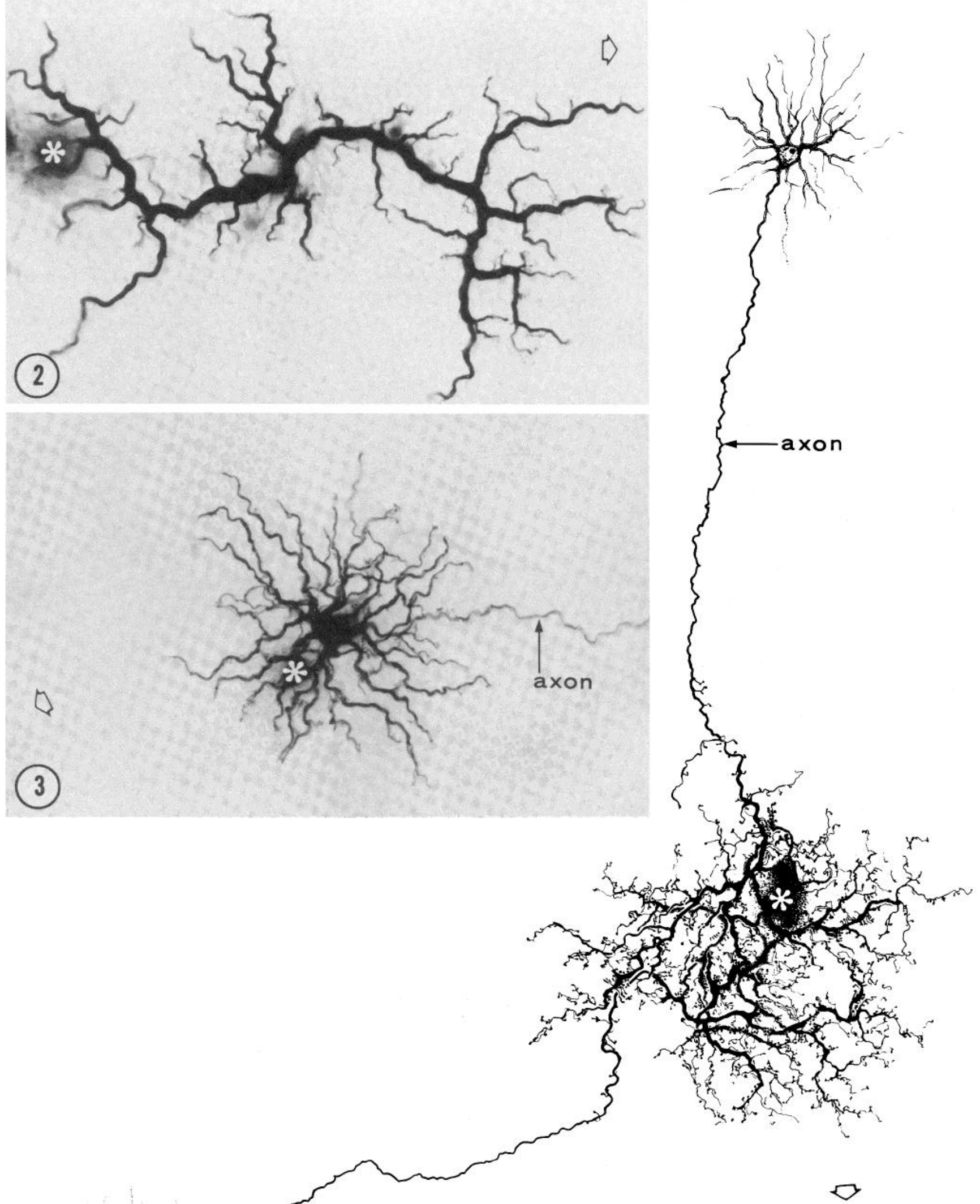
among different cells: on occasion, the transient at the onset of illumination was very prominent and the rod aftereffect was small; in other instances, a small ontransient was associated with a larger rod aftereffect. HRP injections demonstrated that the source of this class of responses was either one type of $\mathrm{HC}$ which lacked an axon (Fig. 2) or the somatic end of a second type of $\mathrm{HC}$ which had an axon and a profuse axonal arborization (Fig. 3). The average input resistance of axonless $\mathrm{HCs}$ was 50 megohms; that of the somatic end of axon-bearing HCs was 70 megohms.

In a second class of responses recorded from $\mathrm{HCs}$, no transient component was observed at stimulus initiation; furthermore, the secondary hyperpolarization after stimulus termination was already present with light intensities of -3.0 log units. With high levels of illumination (-1.0 log unit), the amplitude of the light responses increased only slightly, but the rod aftereffect became equal in amplitude to the primary hyperpolarization and had a prolonged duration, therefore appearing as the dominant component of the response (Fig. 1c). At levels of illumination below $-4.5 \mathrm{log}$ units, the responses were slow and had a latency of $34 \mathrm{msec}$; at stimulus intensities of $-1.0 \mathrm{log}$ unit, the responses remained slow but had a shorter latency of 10 msec. These findings suggest a dominant rod input and a weaker cone influence on the cells penetrated by the microelectrode. HRP injections demonstrated that this class of responses was the property of the axonal arborization of the axon-bearing $\mathrm{HCs}$ (Fig. 4). The average input resistance of the axonal branches was 190 megohms.

Morphology. We stained intracellularly with HRP 50 axonless HCs and 6 somatic ends and 6 axon terminals of axon-bearing HCs. Examination with the light microscope of HRP-injected axonless $\mathrm{HCs}$ showed that they were unusual neurons, having a small number of stout, tortuous dendrites (Fig. 2). These branched sparsely and carried minute, ascending branchlets, each ending with a small swelling. Axonless $\mathrm{HCs}$ were characterized by an extraordinary plcomorphism; some were star-shaped and their dendritic fields were nearly circular or oval with a diameter of about $150 \mu \mathrm{m}$. Other cells were elongate, with dendritic fields measuring $300 \times 75 \mu \mathrm{m}$. Finally, in a third variety of axonless $\mathrm{HC}$, one of the processes was very long and meandered long distances away from the cell body; except for its unusual length, this process was morphologically identical to the other dendrites. The long axis of these cells could reach $350 \mu \mathrm{m}$. Axonless $\mathrm{HCs}$ with a highly asymmetric shape did not exhibit a preferred orientation with respect to the medullary rays. Electron microscopy showed that axonless $\mathrm{HCs}$ contacted the synaptic endings of cone cells; their dendritic branchlets consistently ended as one of the lateral elements of the triads of cone invaginating synapses (Fig. 5).

At the light microscope, we observed that axon-bearing $\mathrm{HCs}$ had a round body which gave rise to numerous, wavy dendrites radiating in all directions (Fig. 3). The dendrites divided sparsely and carried clusters of minute, ascending branchlets slightly swollen at their extremity. The dendritic field was round and had a diameter of 100 to $150 \mu \mathrm{m}$. When HRP was injected into the cell body or one of the dendrites, the axon appeared densely stained at its origin, but it became progressively fainter with increasing distance from the soma. Occasionally, the axon could be followed for 200 to $250 \mu \mathrm{m}$ to the point at which it expanded into its rich, terminal arborization. As a rule, the axon emerged from one of the dendrites and was 0.5 $\mu \mathrm{m}$ thick; its diameter, however, increased near its termination. When HRP was injected into one of the branches of the axonal arborization, the entire cell became filled with the tracer, although the body and dendrites appeared faintly stained (Fig. 4). An interesting observation was that the long axis of this type of $\mathrm{HC}$ was oriented at random with respect to the medullary rays. The tortuous primary branches of the axonal arborization were thicker than the parent axon, reaching a diameter of up to $1 \mu \mathrm{m}$; it was one of those branches which usually was penetrated by the microelectrode. In turn, the primary branches divided profusely, covering a retinal area 200 to $300 \mu \mathrm{m}$ in diameter. All axonal branches were studded with a multitude of tiny ascending telodendria, each ending with a minute knob. Electron microscopy showed that the dendritic branchlets of the axon-bearing HCs contacted cone pedicles; each branchlet consistently ended as one of the lateral elements of the triads of cone invaginating synapses (Fig. 6). On the other hand, the axonal branches contacted rod spherules; each of their

Figure 2. An axonless HC intracellularly injected with HRP. The cell has a small number of stout, tortuous dendrites, which branch sparsely and carry minute, terminal branchlets. At the asterisk, a Müller cell is stained by the small amounts of HRP which leaked from the electrode in the course of the penetration. The open arrow points toward the optic nerve head. This $\mathrm{HC}$ is situated $5.5 \mathrm{~mm}$ below the medullary rays and $1.2 \mathrm{~mm}$ on the nasal side of the vertical meridian. Magnification $\times 550$.

Figure 3. The somatic end of an axon-bearing HC intracellularly injected with HRP. The cell body gives rise to numerous wavy dendrites radiating in all directions; these carry clusters of fine, terminal branchlets. The asterisk indicates the site of electrode penetration. The open arrow points toward the optic nerve head. This $\mathrm{HC}$ is situated $5.2 \mathrm{~mm}$ below the medullary rays and $2 \mathrm{~mm}$ on the temporal side of the vertical meridian. Magnification $\times 550$.

Figure 4. In this experiment, responses typical of the axonal arborization of axon-bearing HCs were recorded both before and after the injection of HRP, but 2 neurons were visualized simultaneously. Perhaps, the electrode moved in the course of the injection from an axonal branch of an axon-bearing $\mathrm{HC}$ to a contiguous axonal branch of another cell of the same type; alternatively, the penetration could have caused the formation of a cytoplasmic bridge between the arborizations of the 2 HCs. The cell bodies and dendrites are faintly stained by the retrograde movement of the tracer. The axons emerge from a dendrite and increase in diameter toward their termination. The primary and secondary branches of the arborization are thicker than the parent axon and divide profusely, carrying a multitude of tiny telodendria, each ending with a minute knob. This camera lucida drawing illustrates two additional, important points: in axon-bearing $\mathrm{HCs}$, the axonal arborizations extensively overlap and the axons do not have a consistent orientation with respect to the medullary rays. The asterisk indicates the site of penetration; the open arrow points toward the optic nerve head. These cells are situated $6 \mathrm{~mm}$ below the medullary rays and $0.7 \mathrm{~mm}$ on the temporal side of the vertical meridian. Magnification $\times 450$. 


telodendria ended as one of the lateral elements of rod invaginating synapses (Fig. 7).

Intercellular dye transfer. The area summation properties of HCs were tested by keeping the stimulus intensity constant at $-3.5 \log$ units and varying the diameter of the illuminating spot. Figure 8 illustrates that both axonless HCs (Fig. 8a) and the somatic end of axonbearing HCs (Fig. $8 b$ ) summated stimuli over a receptive field which was considerably larger than their respective dendritic fields. On the other hand, the receptive field of the axon terminal of axon-bearing HCs (Fig. 8c) was closer in dimensions to its anatomical spread.

We stained intracellularly with LY 3 axonless HCs and 2 somatic ends and 2 axon terminals of axon-bearing HCs. Axonless HCs consistently transferred dye to adjoining, homologous cells (Fig. 9). In one instance, LY spread from the impaled cell to 102 axonless HCs over a circular retinal area $0.9 \mathrm{~mm}$ in diameter. When the somatic end or an axonal branch of the axon-bearing $\mathrm{HCs}$ was injected, LY rapidly spread throughout the entire cell. However, we consistently failed to observe dye transfer to adjoining cells (Figs. 10 and 11). We are confident that our failure was not due to a breakdown of intercellular communication as a consequence of anoxia because, in the same specimen, we observed dye transfer between axonless HCs.

\section{Discussion}

The light responses from rabbit HCs to moderate and high intensity stimuli can be assigned to two basic classes according to $(i)$ the presence of a transient component at stimulus initiation and (ii) the amplitude and duration of a secondary hyperpolarization after stimulus termination. Of these two response characteristics, the transient at the onset of illumination is a property of $\mathrm{HC}$ responses in numerous vertebrate species (Steinberg, 1969b; Gerschenfeld et al., 1980). The secondary hyperpolarizing off-component was described originally in the cat by Steinberg (1969a), who suggested that it was mediated by rods; for this reason, it was termed the rod aftereffect. Later studies in the cat confirmed both its existence and its rod origin (Nelson et al., 1976; Nelson, 1977). Also in the rabbit, a rod aftereffect was demonstrated in HC responses (De Monasterio, 1978; Dacheux and Miller, 1981a); Dacheux and Miller (1981a) reported that adaptation with rod-matched chromatic light exerted little influence on the sustained component of the response or on the fast repolarization at stimulus termination but that it reduced the amplitude and duration of the rod aftereffect. Therefore, a ratio of the cone-to-rod influence on $\mathrm{HC}$ responses was deduced from the relative amplitude of the fast and slow components in the repo-

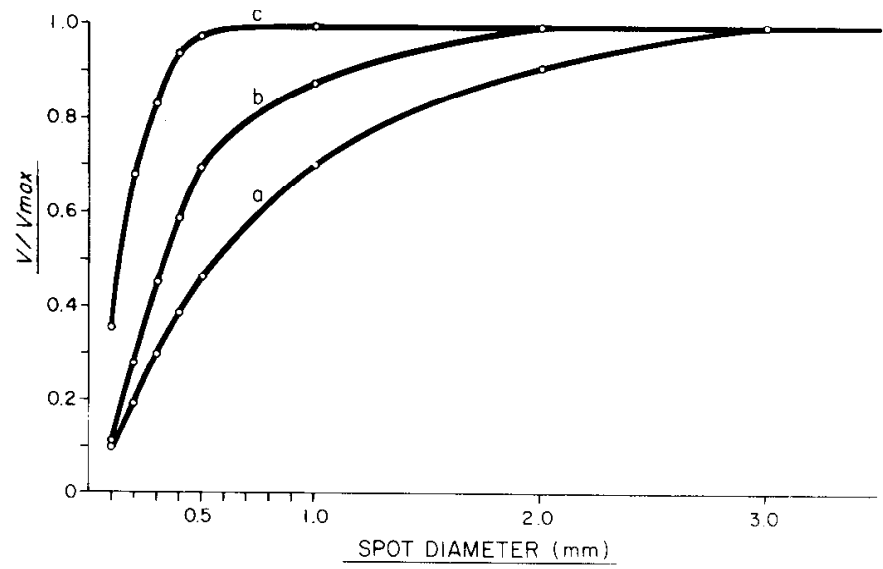

Figure 8. The area summation properties of rabbit $\mathrm{HCs}$ were investigated by keeping the stimulus intensity constant and varying the diameter of the illuminating spot. The normalized amplitude of the averaged responses from 10 cells is shown on the ordinate. The diagram demonstrates that the diameter of the receptive field measures about $3 \mathrm{~mm}$ for axonless HCs $(a)$, $2 \mathrm{~mm}$ for the somatic end of axon-bearing HCs $(b)$, and $0.5 \mathrm{~mm}$ for the axonal arborization of axon-bearing HCs $(c)$.

larization at the end of high intensity stimuli. In the present paper, this cone-to-rod index is the basis for separating rabbit $\mathrm{HC}$ responses inlo two classes.

We have now identified morphologically the source of these classes of responses. Axonless $\mathrm{HCs}$ and the somatic end of the axon-bearing $\mathrm{HCs}$ share similar response features to illumination with white light, namely a transient superimposed on a sustained hyperpolarization at stimulus initiation and a smaller, secondary hyperpolarization al the cessation of high intensity stimuli. Both are connected to cones but also receive rod influence; both summate stimuli from an area which is much larger than their respective dendritic fields. The axonal arborization of the axon-bearing HCs exhibits response properties different from those of the cell body: it has no transient at stimulus initiation and the secondary hyperpolarization at high light intensities is very prominent. It is connected to rods but also receives cone influence. Its receptive field approximates in diameter the anatomical spread of its branches.

It has been stated that electrotonic decay along the thin and long axon of mammalian axon-bearing HCs prevents the transfer of information from the body to the axon terminal and vice versa (Nelson et al., 1975); this may explain why the somatic end has response properties different from those of the axon terminal. We do not know the source of the dual receptor influence on axonless HCs and the somatic end and axonal arborization of axon-bearing HCs; signals may spill over though the gap

Figure 5. An axonless HC was injected with HRP intracellularly and prepared for electron microscopy. The dendritic branchlets of the stained cell (asterisks) end as one of the lateral elements of the triad in four invaginating synapses of a cone pedicle. Note that the lateral elements of each invaginating synapse belong to 2 different HCs. $R b$, Synaptic ribbons. Magnification $\times 19,000$.

Figure 6. Two dendritic branchlets of an HRP-injected, axon-bearing HC (asterisks) end as one of the lateral elements of the triad in two invaginating synapses of a cone pedicle. Note that the lateral elements of each invaginating synapse belong to 2 different HCs. $R b$, Synaptic ribbons. Magnification $\times 46,000$.

Figure 7. The telodendron of an HRP-injected axon terminal of an axon-bearing HC (asterisk) ends as one of the lateral elements in the invaginating synapse of a rod spherule. The spherule is connected to an adjacent cone pedicle by a gap junction (arrowhead). $R b$, Synaptic ribbon. Magnification $\times 29,500$. 

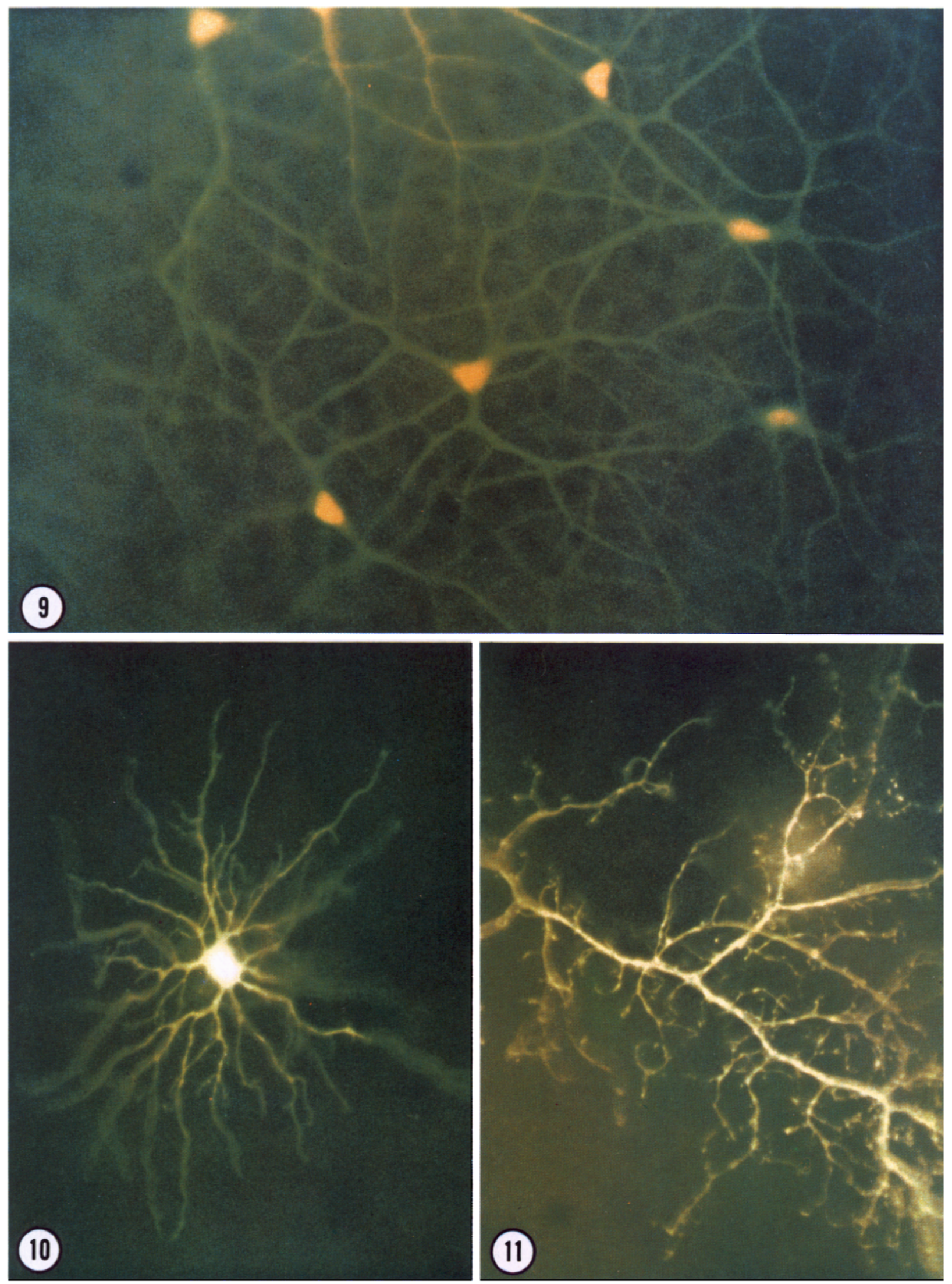

Figure 9. Axonless HCs exchange intracellularly injected LY. Note that the fluorescent dye has a strong affinity for the cell nuclei. Magnification $\times 500$.

Figure 10. In the somatic end of axon-bearing HCs, we failed to demonstrate the transfer of intracellularly injected LY to adjacent cells. Magnification $\times 600$.

Figure 11. Also in the axon terminal of axon-bearing HCs, we failed to demonstrate the transfer of LY to adjacent cells. Magnification $\times 750$. 
junctions which connect rod to cone cells (Raviola and Gilula, 1973). However, we must first exclude, by serial sectioning, the presence of synaptic connections with both types of photoreceptors. Further work is also necessary to clarify the mechanism underlying the variations in the response profile of axonless $\mathrm{HCs}$ and the somatic end of axon-bearing HCs. Dacheux and Miller (1981a) suggested that axonless $\mathrm{HCs}$ have a larger on-transient and smaller rod aftereffect than the somatic end of axonbearing HCs; this seems to hold true for many of the cells from which we recorded. However, among axonless HCs, we found marked fluctuations in the amplitude of these response components. It is unclear whether this discrepancy between the present and previous results reflects differences in the functional state of the cells, in the region of the cell sampled by the microelectrode, or in the pattern of connections with the photoreceptors. Finally, the question emerges: why in the rod-dominant rabbit retina are both axonless HCs and the somatic end of axon-bearing HCs connected to cones? Analysis of the chromatic input into these neurons may solve this crucial problem.

The waveforms of the different responses of rabbit $\mathrm{HCs}$ to diffuse white light stimulation closely resemble those reported in the cat (Nelson et al., 1976; Nelson, 1977). Furthermore, the cells in the two species share similar connections with the photoreceptors (Kolb, 1974). A striking difference, however, exists in the shape of axonless HCs: some of them have a circular dendritic field as in the cat, whereas others are highly asymmetric. On the other hand, the morphology of axon-bearing $\mathrm{HCs}$ does not differ significantly in the two species.

The dramatic transfer of fluorescent dye among axonless HCs explains why their receptive fields are much larger than their dendritic spread: these cells are connected by extensive gap junctions (Raviola and Gilula, 1975) which are permeable to the low molecular weight probe. On the other hand, it is unclear why there is a discrepancy between the size of the receptive field and the diameter of the dendritic tree in the somatic end of axon-bearing $\mathrm{HCs}$, for we failed to demonstrate intercellular dye transfer. It is known, however, that certain cell types possess gap junctions and exhibit ionic coupling but do not exchange fluorescent probes (Lo and Gilula, 1979). Possibly, the gap junctions are few or small and the fluorescence of the minute amount of dye which has moved to adjacent cells is quenched during observation. Alternatively, these cells may uncouple as a result of the disturbance of the internal milieu caused by the tracer injection. A different situation may exist in the axonal ending of the axon-bearing HCs: it does not transfer dye, but the difference between the diameter of its receptive field and its anatomic spread is considerably smaller than in the somatic end. This discrepancy could well be caused by light scattering or the effect of electrical coupling between the receptors.

\section{References}

Adams, J. C. (1977) Technical considerations on the use of horseradish peroxidase as a neuronal marker. Neuroscience
2: $141-145$.

Boycott, B. B., and H. Kolb (1973) The connections between bipolar cells and photoreceptors in the retina of the domestic cat. J. Comp. Neurol. 148: 91-114.

Dacheux, R. F., and R. F. Miller (1981a) An intracellular electrophysiological study of the ontogeny of functional synapses in the rabbit retina. I. Receptors, horizontal, and bipolar cells. J. Comp. Neurol. 198: 307-326.

Dacheux, R. F., and R. F. Miller (1981b) An intracellular electrophysiological study of the ontogeny of functional synapses in the rabbit retina. II. Amacrine cells. J. Comp. Neurol. 198: 327-334.

De Monasterio, F. M. (1978) Spectral interactions in horizontal and ganglion cells of the isolated and arterially-perfused rabbit retina. Brain Res. 150: 239-258.

Dowling, J. E., and B. B. Boycott (1966) Organization of the primate retina: Electron microscopy. Proc. R. Soc. Lond. (Biol.) 166: 80-111.

Gerschenfeld, H. M., M. Piccolino, and J. Neyton (1980) Feedback modulation of cone synapses by L-horizontal cells of turtle retina. J. Exp. Biol. 89: 177-192.

Karnovsky, M. J. (1971) Use of ferrocyanide-reduced osmium tetroxide in electron microscopy. In Proceedings of the 11th Annual Meeting of the American Society for Cell Biology, 146 (Abstr.), New Orleans.

Kolb, H. (1970) Organization of the outer plexiform layer of the primate retina: Electron microscopy of Golgi-impregnated cells. Philos. Trans. R. Soc. Lond. (Biol.) 258: 261-283.

Kolb, H. (1974) The connections between horizontal cells and photoreceptors in the retina of the cat: Electron microscopy of Golgi preparations. J. Comp. Neurol. 155: 1-14.

Kolb, H. (1977) The organization of the outer plexiform layer in the retina of the cat: Electron microscopic observations. J. Neurocytol. 6: 131-153.

Lo, C. W., and N. B. Gilula (1979) Gap junctional communication in the post-implantation mouse embryo. Cell 18: 411-422.

Miller, R. F., and R. F. Dacheux (1973) Information processing in the retina: Importance of chloride ions. Science 181: 266-268.

Missotten, L. (1965) The Ultrastructure of the Human Retina, Editions Arscia Uitgaven, Brussels.

Nelson, R. (1977) Cat cones have rod input: A comparison of the response properties of cones and horizontal cell bodies in the retina of the cat. J. Comp. Neurol. 172: 109-136.

Nelson, R., A. von Lutzow, H. Kolb, and P. Gouras (1975) Horizontal cells in cat retina with independent dendritic systems. Science 189: 137-139.

Nelson, R., H. Kolb, E. V. Famiglietti, Jr., and P. Gouras (1976) Neural responses in the rod and cone systems of the cat retina: Intracellular records and Procion stains. Invest. Ophthalmol. 15: 946-953.

Raviola, E., and N. B. Gilula (1973) Gap junctions between photoreceptor cells in the vertebrate retina. Proc. Natl. Acad. Sci. U. S. A. 70: 1677-1681.

Raviola, E., and N. B. Gilula (1975) Intramembrane organization of specialized contacts in the outer plexiform layer of the retina. A freeze-fracture study in monkeys and rabbits. J. Cell Biol. 65: 192-222.

Steinberg, R. H. (1969a) The rod after-effect in S-potentials from the cat retina. Vision Res. 9: 1345-1355.

Steinberg, R. H. (1969b) Rod-cone interactions in S-potentials from the cat retina. Vision Res. 9: 1331-1344.

Stewart, W. W. (1978) Functional connections between cells as revealed by dye coupling with a highly fluorescent naphthalimide tracer. Cell 14: 741-759. 